\title{
Worst-Case Optimal Adaptive Prefix Coding
}

\author{
Travis Gagie ${ }^{1, \star}$ and Yakov Nekrich ${ }^{2}$ \\ Research Group in Genome Informatics \\ University of Bielefeld, Germany \\ travis.gagie@gmail.com \\ Department of Computer Science \\ University of Bonn, Germany \\ yasha@cs.uni-bonn.de
}

\begin{abstract}
A common complaint about adaptive prefix coding is that it is much slower than static prefix coding. Karpinski and Nekrich recently took an important step towards resolving this: they gave an adaptive Shannon coding algorithm that encodes each character in $O(1)$ amortized time and decodes it in $O(\log H)$ amortized time, where $H$ is the empirical entropy of the input string $s$. For comparison, Gagie's adaptive Shannon coder and both Knuth's and Vitter's adaptive Huffman coders all use $\Theta(H)$ amortized time for each character. In this paper we give an adaptive Shannon coder that both encodes and decodes each character in $O(1)$ worst-case time. As with both previous adaptive Shannon coders, we store $s$ in at most $(H+1)|s|+o(|s|)$ bits. We also show that this encoding length is worst-case optimal up to the lower order term.
\end{abstract}

\section{Introduction}

Adaptive prefix coding is a well studied problem whose well known and widely used solution, adaptive Huffman coding, is nevertheless not worst-case optimal. Suppose we are given a string $s$ of length $m$ over an alphabet of size $n$. For static prefix coding, we are allowed to make two passes over $s$ but, after the first pass, we must build a single prefix code, such as a Shannon code [16] or Huffman code [9], and use it to encode every character. Since a Huffman code minimizes the expected codeword length, static Huffman coding is optimal (ignoring the asymptotically negligible $O(n \log n)$ bits needed to write the code). For adaptive prefix coding, we are allowed only one pass over $s$ and must encode each character with a prefix code before reading the next one, but we can change the code after each character. Assuming we compute each code deterministically from the prefix of $s$ already encoded, we can later decode $s$ symmetrically. The most intuitive solution is to encode each character using a Huffman code for the prefix already encoded; Knuth [11] showed how to do this in time proportional to the

\footnotetext{
* This paper was written while the second author was at the University of Eastern Piedmont, Italy, supported by Italy-Israel FIRB Project "Pattern Discovery Algorithms in Discrete Structures, with Applications to Bioinformatics".
} 
length of the encoding produced, taking advantage of a property of Huffman codes discovered by Faller [3] and Gallager [7]. Shortly thereafter, Vitter [18] gave another adaptive Huffman coder that also uses time proportional to the encoding's length; he proved his coder stores $s$ in fewer than $m$ more bits than static Huffman coding, and that this is optimal for any adaptive Huffman coder. With a similar analysis, Milidiú, Laber and Pessoa [13] later proved Knuth's coder uses fewer than $2 m$ more bits than static Huffman coding. In other words, Knuth's and Vitter's coders store $s$ in at most $(H+2+h) m+o(m)$ and $(H+$ $1+h) m+o(m)$ bits, respectively, where $H=\sum_{a}(\operatorname{occ}(a, s) / m) \log (m / \operatorname{occ}(a, s))$ is the empirical entropy of $s$ (i.e., the entropy of the normalized distribution of characters in $s), \operatorname{occ}(a, s)$ is the number of occurrences of the character $a$ in $s$, and $h \in[0,1)$ is the redundancy of a Huffman code for $s$; therefore, both adaptive Huffman coders use $\Theta(H)$ amortized time to encode and decode each character of $s$. Turpin and Moffat [17] gave an adaptive prefix coder that uses canonical codes, and showed it achieves nearly the same compression as adaptive Huffman coding but runs much faster in practice. Their upper bound was still $O(H)$ amortized time for each character but their work raised the question of asymptotically faster adaptive prefix coding. In all of the above algorithms the encoding and decoding times are proportional to the bit length of the encoding. This implies that we need $O(H)$ time to encode/decode each symbol; since entropy $H$ depends on the size of the alphabet, the running times grow with the alphabet size.

The above results for adaptive prefix coding are in contrast to the algorithms for the prefix coding in the static scenario. The simplest static Huffman coders use $\Theta(H)$ amortized time to encode and decode each character but, with a lookup table storing the codewords, it is not hard to speed up encoding to take $O(1)$ worst-case time for each character. We can also decode an arbitrary prefix code in $O(1)$ time using a look-up table, but the space usage and initialization time for such a table can be prohibitively high, up to $O(m)$. Moffat and Turpin [14] described a practical algorithm for decoding prefix codes in $O(1)$ time; their algorithm works for a special class of prefix codes, the canonical codes introduced by Schwartz and Kallick [15].

While all adaptive coding methods described above maintain the optimal Huffman code, Gagie [6] described an adaptive prefix coder that is based on sub-optimal Shannon coding; his method also needs $O(H)$ amortized time per character for both encoding and decoding. Although the algorithm of [6] maintains a Shannon code that is known to be worse than the Huffman code in the static scenario, it achieves $(H+1) m+o(m)$ upper bound on the encoding length that is better than the best known upper bounds for adaptive Huffman algorithms. Karpinski and Nekrich [10] recently reduced the gap between static and adaptive prefix coding by using quantized canonical coding to speed up an adaptive Shannon coder of Gagie [6]: their coder uses $O(1)$ amortized time to encode each character and $O(\log H)$ amortized time to decode it; the encoding length is also at most $(H+1) m+o(m)$ bits.

In this paper we describe an algorithm that both encodes and decodes each character in $O(1)$ worst-case time, while still using at most $(H+1) m+o(m)$ 
bits. It can be shown that the encoding length of any adaptive prefix coding algorithm is $(H+1) m-o(m)$ bits in the worst case. Thus our algorithm works in optimal worst-case time independently of the alphabet size and achieves optimal encoding length (up to the lower-order term). As is common, we assume $n \ll m^{1}$; for simplicity, we also assume $s$ contains at least two distinct characters and $m$ is given in advance. Our model is a unit-cost word RAM with $\Omega(\log m)$-bit words on which it takes $O(1)$ time to input or output a word. Our encoding algorithm uses only addition and bit operations; our decoding algorithm also uses multiplication and finding the most significant bit in $O(1)$ time. We can also implement the decoding algorithm, so that it uses $A C^{0}$ operations only. Encoding needs $O(n)$ words of space, and decoding needs $O(n \log m)$ words of space. The decoding algorithm can be implemented with bit operations only at a cost of higher space usage and additional pre-processing time. For an arbitrary constant $\alpha>0$, we can construct in $O\left(m^{\alpha}\right)$ time a look-up table that uses $O\left(m^{\alpha}\right)$ space; this look-up table enables us to implement multiplications with $O(1)$ table look-ups and bit operations.

While the algorithm of [10] uses quantized coding, i.e., coding based on the quantized symbol probabilities, our algorithm is based on delayed probabilities: encoding of a symbol $s[i]$ uses a Shannon code for the prefix $s[1 . . i-d]$ for an appropriately chosen parameter $d$; henceforth $s[i]$ denotes the $i$-th symbol in the string $s$ and $s[i . . j]$ denotes the substring of $s$ that consists of symbols $s[i] s[i+1] \ldots s[j]$. In Section 2 we describe canonical Shannon codes and explain how they can be used to speed up Shannon coding. In Section 3 we describe two useful data structures that allow us to maintain the Shannon code efficiently. We present our algorithm and analyze the number of bits it needs to encode a string in Section 4. In Section 5 we prove a matching lower bound by extending Vitter's lower bound from adaptive Huffman coders to all adaptive prefix coders. In section 6 we show that our technique can be applied to the online stable sorting problem. In section 7 we describe how we can use the same approach of delayed adaptive coding to achieve $O(1)$ worst-case encoding time for several other coding problems in the adaptive scenario.

\section{Canonical Shannon coding}

Shannon [16] defined the entropy $H(P)$ of a probability distribution $P=p_{1}, \ldots, p_{n}$ to be $\sum_{i=1}^{n} p_{i} \log _{2}\left(1 / p_{i}\right) .^{2}$ He then proved that, if $P$ is over an alphabet, then we can assign each character with probability $p_{i}>0$ a prefix-free binary codeword of length $\left\lceil\log _{2}\left(1 / p_{i}\right)\right\rceil$, so the expected codeword length is less than $H(P)+1$; we cannot, however, assign them codewords with expected length less than $H(P) .^{3}$

\footnotetext{
${ }^{1}$ In fact, our main result is valid if $n=o\left(m / \log ^{5 / 2} m\right)$. For the result of section 6 and two results in section 7 we need a somewhat stronger assumption that $n=$ $o(\sqrt{m / \log m})$

${ }^{2}$ We assume $0 \log (1 / 0)=0$.

${ }^{3}$ In fact, these bounds hold for any size of code alphabet; we assume throughout that codewords are binary, and by log we always mean $\log _{2}$.
} 
Shannon's proof of his upper bound is simple: without loss of generality, assume $p_{1} \geq \cdots \geq p_{n}>0$; for $1 \leq i \leq n$, let $b_{i}=\sum_{j=1}^{i-1} p_{j}$; since $\left|b_{i}-b_{i^{\prime}}\right| \geq p_{i}$ for $i^{\prime} \neq i$, the first $\left\lceil\log \left(1 / p_{i}\right)\right\rceil$ bits of $b_{i}$ 's binary representation uniquely identify it; let these bits be the codeword for the $i$ th character. The codeword lengths do not change if, before applying Shannon's construction, we replace each $p_{i}$ by $1 / 2^{\left\lceil\log \left(1 / p_{i}\right)\right\rceil}$. The code then produced is canonical [15]: i.e., if a codeword is the $c$ th of length $r$, then it is the first $r$ bits of the binary representation of $\sum_{\ell=1}^{r-1} W(\ell) / 2^{\ell}+(c-1) / 2^{r}$, where $W(\ell)$ is the number of codewords of length $\ell$. For example,
1) 000
7) 1000
2) 001
8) 1001
3) 0100
9) 10100
4) 0101
5) 0110
10) 10101
6) 0111
16) 11011

are the codewords of a canonical code. Notice the codewords are always in lexicographic order.

Static prefix coding with a Shannon code stores $s$ in $(H+1) m+o(m)$ bits. An advantage to using a canonical Shannon code is that we can easily encode each character in $O(1)$ worst-case time (apart from first pass) and decode it symmetrically in $O(\log \log m)$ worst-case time (see [14]). To encode a symbol $s[i]$ from $s$, it suffices to know the pair $\langle r, c\rangle$ such that the codeword for $s[i]$ is the $c$-th codeword of length $r$, and the first codeword $l_{r}$ of length $r$. Then the codeword for $s[i]$ can be computed in $O(1)$ time as $l_{r}+c$. We store the pair $\langle r, c\rangle$ for the $k$-th symbol in the alphabet in the $k$-th entry of the array $C$. The array $L[1 . .\lceil\log m\rceil]$ contains first codewords of length $l$ for each $1 \leq l \leq\lceil\log m\rceil$. Thus, if we maintain arrays $L$ and $C$ we can encode a character from $s$ in $O(1)$ time.

For decoding, we also need a data structure $D$ of size $\log m$ and a matrix $M$ of size $n \times\lceil\log m\rceil$. For each $l$ such that there is at least one codeword of length $l$, the data structure $D$ contains the first codeword of length $l$ padded with $\lceil\log m\rceil-l$ 0's. For an integer $q, D$ can find the predecessor of $q$ in $D$, $\operatorname{pred}(q, D)=\max \{x \in D \mid x \leq q\}$. The entry $M[r, c]$ of the matrix $M$ contains the symbol $s$, such that the codeword for $s$ is the $c$-th codeword of length $r$. The decoding algorithm reads the next $\lceil\log m\rceil$ bits into a variable $w$ and finds the predecessor of $w$ in $D$. When $\operatorname{pred}(w, D)$ is known, we can determine the length $r$ of the next codeword, and compute its index $c$ as $(w-L[r]) \gg(\lceil\log m\rceil-r)$ where $\gg$ denotes the right bit shift operation.

The straightforward binary tree solution allows us to find predecessors in $O(\log \log m)$ time. We will see in the next section that predecessor queries on a set of $\log m$ elements can be answered in $O(1)$ time. Hence, both encoding and decoding can be performed in $O(1)$ time in the static scenario. In the adaptive scenario, we must find a way to maintain the arrays $C$ and $L$ efficiently and, in the case of decoding, the data structure $D$. 


\section{Data structures}

It is not hard to speed up the method for decoding we described in Section 2. For example, if the augmented binary search tree we use as $D$ is optimal instead of balanced then, by Jensen's Inequality, we decode each character in $O(\log H)$ amortized time. Even better, if we use a data structure by Fredman and Willard [4], then we can decode each character in $O(1)$ worst-case time.

Lemma 1 (Fredman and Willard, 1993). Given $O\left(\log ^{1 / 6} m\right)$ keys, in $O\left(\log ^{2 / 3} m\right)$ worst-case time we can build a data structure that stores those keys and supports predecessor queries in $O(1)$ worst-case time.

Corollary 1. Given $O(\log m)$ keys, in $O\left(\log ^{3 / 2} m\right)$ worst-case time we can build a data structure that stores those keys and supports predecessor queries in $O(1)$ worst-case time.

Proof. We store the keys in the leaves of a search tree with degree $O\left(\log ^{1 / 6} m\right)$, size $O\left(\log ^{5 / 6} \mathrm{~m}\right)$ and height at most 5 . Each node stores an instance of Fredman and Willard's data structure from Lemma 1: each data structure associated with a leaf stores $O\left(\log ^{1 / 6} m\right)$ keys and each data structure associated with an internal node stores the first key in each of its children's data structures. It is straightforward to build the search tree in $O\left(\log ^{2 / 3+5 / 6} m\right)=O\left(\log ^{3 / 2} m\right)$ time and implement queries in $O(1)$ time.

In Lemma 1 and Corollary 1 we assume that multiplication and finding the most significant bit of an integer can be performed in constant time. As shown in [1], we can implement the data structure of Lemma 1 using $A C^{0}$ operations only. We can restrict the set of elementary operations to bit operations and table look-ups by increasing the space usage and preprocessing time to $O\left(m^{\varepsilon}\right)$. In our case all keys in the data structure $D$ are bounded by $m$; hence, we can construct in $O\left(m^{\varepsilon}\right)$ time a look-up table that uses $O\left(m^{\varepsilon}\right)$ space and allows us to multiply two integers or find the most significant bit of an integer, in constant time.

Corollary 1 is useful to us because the data structure it describes not only supports predecessor queries in $O(1)$ worst-case time but can also be built in time polylogarithmic in $m$; the latter property will let our adaptive Shannon coder keep its data structures nearly current by regularly rebuilding them. The array $C$ and matrix $M$ cannot be built in $o(n)$ time, however, so we combine them in a data structure that can be updated incrementally. Arrays $C[]$ and $L[]$, and the matrix $M$ defined in the previous section, can be rebuilt as described in the next Lemma.

Lemma 2. If codeword lengths of $f \geq \log m$ symbols are changed, we can rebuild arrays $C[]$ and $L[]$, and update the matrix $M$ in $O(f)$ time.

Proof. We maintain an array $S$ of doubly-linked lists. The doubly-linked list $S[l]$ contains all symbols with codeword length $l$ sorted by their codeword indices, i.e, the codeword of the $c$-th symbol in array $S[r]$ is the $c$-th codeword of length 
$r$. The number of codewords with lengths $l$ for each $1 \leq l \leq\lceil\log m\rceil$ is stored in the array $W[]$. If the codeword length of a symbol $a$ is changed from $l_{1}$ to $l_{2}$, we replace $a$ with last $\left[l_{1}\right]$ in $S\left[l_{1}\right]$ and append $a$ at the end of $S\left[l_{2}\right]$. Then, we set $C\left[k_{l}\right]=C\left[k_{a}\right]$ and $C\left[k_{a}\right]=\left\langle l_{2}, W\left[l_{2}\right]\right\rangle$, where $k_{a}$ and $k_{l}$ are indices of $a$ and last $\left[l_{1}\right]$ in the array $C[]$. Finally, we increment $W\left[l_{2}\right]$, decrement $W\left[l_{1}\right]$ and update last $\left[l_{1}\right]$ and last $\left[l_{2}\right]$. We also update entries $M\left[l_{1}, c_{a}\right], M\left[l_{1}, W\left[l_{1}\right]-1\right]$ and $M\left[l_{2}, W\left[l_{2}\right]\right]$ in the matrix $M$ accordingly, where $c_{a}$ is the index of $a$ 's codeword before the update operation. Thus the array $C$ and the matrix $M$ can be updated in $O(1)$ time when the codeword length of a symbol is changed.

When codeword lengths of all $f$ symbols are changed, we can compute the array $L$ from scratch in $O(\log m)=O(f)$ time.

\section{Algorithm}

The main idea of the algorithm of [10], that achieves $O(1)$ amortized encoding cost per symbol, is quantization of probabilities. The Shannon code is maintained for the probabilities $\tilde{p}_{j}=\frac{\lceil i / q]}{\left\lfloor\operatorname{occ}\left(a_{i}, s[1 . . i]\right) / q\right]}$ where occ $\left(a_{j}, s[1 . . i]\right)$ denotes the number of occurrences of the symbol $a_{j}$ in the string $s[1 . . i]$ and the parameter $q=\Theta(\log m)$. The symbol $a_{i}$ must occur $q$ times before the denominator of the fraction $\tilde{p}_{i}$ is incremented by 1 . Roughly speaking, the value of $\tilde{p}_{i}$, and hence the codeword length of $a_{i}$, changes at most once after $\log m$ occurrences of $a_{i}$. As shown in Lemma 2, we can rebuild the arrays $C[]$ and $L[]$ in $O(\log m)$ time. Therefore encoding can be implemented in $O(1)$ amortized time. However, it is not clear how to use this approach to obtain constant worst-case time per symbol.

In this paper a different approach is used. Symbols $s[i+1], s[i+2], \ldots, s[i+d]$ are encoded with a Shannon code for the prefix $s[1] s[2] \ldots s[i-d]$ of the input string. Recall that in a traditional adaptive code the symbol $s[i+1]$ is encoded with a code for $s[1] \ldots s[i]$. While symbols $s[i+1] \ldots s[i+d]$ are encoded, we build an optimal code for $s[1] \ldots s[i]$. The next group of symbols, i.e. $s[i+d+$ $1] \ldots s[i+2 d]$ will be encoded with a Shannon code for $s[1] \ldots s[i]$, and the code for $s[1] \ldots s[i+d]$ will be simultaneously rebuilt in the background. Thus every symbol $s[j]$ is encoded with a Shannon code for the prefix $s[1] \ldots s[j-t]$, $d \leq t<2 d$, of the input string. That is, when a symbol $s[i]$ is encoded, its codeword length equals

$$
\left\lceil\log \frac{i+n-t}{\max (\operatorname{occ}(s[i], s[1 . . i-t]), 1)}\right\rceil .
$$

We increased the enumerator of the fraction by $n$ and the denominator is always at least 1 because we assume that every character is assigned a codeword of length $\lceil\log n-d\rceil$ before encoding starts. We make this assumption only to simplify the description of our algorithm. There are others methods of dealing with characters that occur for the first time in the input string that are more practically efficient, see e.g. [11]. The method of [11] can also be used in our algorithm, but it would not change the total encoding length. 
Later we will show that the delay of at most $2 d$ increases the length of encoding only by a lower order term. Now we turn to the description of the procedure that updates the code, i.e. we will show how the code for $s[1] \ldots s[i]$ can be obtained from the code for $s[1] \ldots s[i-d]$.

Let $\mathcal{C}$ be an optimal code for $s[1] \ldots s[i-d]$ and $\mathcal{C}^{\prime}$ be an optimal code for $s[1] \ldots s[i]$. As shown in section 2, updating the code is equivalent to updating the arrays $C[]$ and $L[]$, the matrix $M$, and the data structure $D$. Since a group of $d$ symbols contains at most $d$ different symbols, we must change codeword lengths of at most $d$ codewords. The list of symbols $a_{1}, \ldots, a_{k}$ such that the codeword length of $a_{k}$ must be changed can be constructed in $O(d)$ time. We can construct an array $L[]$ for the code $\mathcal{C}^{\prime}$ in $O(\max (d, \log m))$ time by Lemma 2 . The matrix $M$ and the array $C[]$ can be updated in $O(d)$ time because only $O(d)$ cells of $M$ are modified. However, we cannot build new versions of $M$ and $C[]$ because they contain $\Theta(n \log m)$ and $\Theta(n)$ cells respectively. Since we must obtain the new version of $M$ while the old version is still used, we modify $M$ so that each cell of $M$ is allowed to contain two different values, an old one and a new one. For each cell $(r, c)$ of $M$ we store two values $M[r, c] . o l d$ and $M[r, c] . n e w$ and the separating value $M[r, c] . b$ : when the symbol $s[t], t<M[r, c] . b$, is decoded, we use $M[r, c] . o l d$; when the symbol $s[t], t \geq M[r, c] . b$, is decoded, we use $M[r, c] . n e w$. The procedure for updating $M$ works as follows: we visit all cells of $M$ that were modified when the code $\mathcal{C}$ was constructed. For every such cell we set $M[r, c]$. old $=M[r, c]$. new and $M[r, c] . b=+\infty$. Then, we add the new values for those cells of $M$ that must be modified. For every cell that must be modified, the new value is stored in $M[r, c]$.new and $M[r, c] . b$ is set to $i+d$. The array $C[]$ can be updated in the same way. When the array $L[]$ is constructed, we can construct the data structure $D$ in $O\left(\log ^{3 / 2} m\right)$ time.

The algorithm described above updates the code if the codeword lengths of some of the symbols $s[i-d+1] \ldots s[i]$ are changed. But if some symbol $a$ does not occur in the substring $s[i-d+1] \ldots s[i]$, its codeword length might still change in the case when $\log (i)>\log \left(i_{a}\right)$ where $i_{a}=\max \{j<i \mid s[j]=a\}$. We can, however, maintain the following invariant on the codeword length $l_{a}$ :

$$
\left\lceil\log \frac{i+n}{\max (\operatorname{occ}(a, s[1 . . i-2 d]), 1)}\right\rceil \leq l_{a} \leq\left\lceil\log \frac{i+2 n}{\max (\operatorname{occ}(a, s[1 . . i-2 d]), 1)}\right\rceil .
$$

When the codeword length of a symbol $a$ must be modified, we set its length to $\left\lceil\log \frac{i+2 n}{\max (\operatorname{occ}(a, s[1 . i]), 1)}\right\rceil$. All symbols $a$ are also stored in the queue $Q$. When the code $\mathcal{C}^{\prime}$ is constructed, we extract the first $d$ symbols from $Q$, check whether their codeword lengths must be changed, and append those symbols at the end of $Q$. Thus the codeword length of each symbol is checked at least once when an arbitrary substring $s[u] \ldots s[u+n]$ of the input string $s$ is processed. Clearly, the invariant 1 is maintained.

Thus the procedure for obtaining the code $\mathcal{C}^{\prime}$ from the code $\mathcal{C}$ consists of the following steps: 
1. check symbols $s[i-d+1] \ldots s[i]$ and the first $d$ symbols in the queue $Q$; construct a list of codewords whose lengths must be changed; remove the first $d$ symbols from $Q$ and append them at the end of $Q$

2. traverse the list $\mathcal{N}$ of modified cells in the matrix $M$ and the array $C[]$, and remove the old values from those cells; empty the list $\mathcal{N}$

3. update the array $C[]$ for code $\mathcal{C}^{\prime}$; simultaneously, update the matrix $M$ and construct the list $\mathcal{N}$ of modified cells in $M$ and $C[]$

4. construct the array $L$ and the data structure $D$ for the new code $\mathcal{C}^{\prime}$

Each of the steps described above, except the last one, can be performed in $O(d)$ time; the last step can be executed in $O\left(\max \left(d, \log ^{3 / 2} m\right)\right)$ time. For $d=$ $\left\lfloor\log ^{3 / 2} m\right\rfloor / 2$, code $\mathcal{C}$ can be constructed in $O(d)$ time. If the cost of constructing $\mathcal{C}^{\prime}$ is evenly distributed among symbols $s[i-d], \ldots, s[i]$, then we spend $O(1)$ extra time when each symbol $s[j]$ is processed. Since occ $\left(a, s\left[1 . . i-\left\lfloor\log ^{3 / 2} m\right\rfloor\right]\right) \geq$ $\max \left(\operatorname{occ}(a, s[1 . . i])-\left\lfloor\log ^{3 / 2} m\right\rfloor, 1\right)$, we need at most

$$
\left\lceil\log \frac{i+2 n}{\max \left(\operatorname{occ}(s[i], s[1 . . i])-\left\lfloor\log ^{3 / 2} m\right\rfloor, 1\right)}\right\rceil
$$

bits to encode $s[i]$.

Lemma 3. We can keep an adaptive Shannon code such that, for $1 \leq i \leq m$, the codeword for $s[i]$ has length at most

$$
\left\lceil\log \frac{i+2 n}{\max \left(\operatorname{occ}(s[i], s[1 . . i])-\left\lfloor\log ^{3 / 2} m\right\rfloor, 1\right)}\right\rceil
$$

and we use $O(1)$ worst-case time to encode and decode each character.

Gagie [6] and Karpinski and Nekrich [10] proved inequalities that, together with Lemma 3, immediately yield our result.

Theorem 1. We can encode $s$ in at most $(H+1) m+o(m)$ bits with an adaptive prefix coding algorithm that encodes and decodes each character in $O(1)$ worstcase time.

For the sake of completeness, we summarize and prove their inequalities as the following lemma:

Lemma 4. $\sum_{i=1}^{m}\left\lceil\log \frac{i+2 n}{\max \left(\operatorname{occ}(s[i], s[1 . . i])-\left\lfloor\log ^{3 / 2} m\right\rfloor, 1\right)}\right\rceil \leq(H+1) m+O\left(n \log ^{5 / 2} m\right)$. 
Proof. Let

$$
\begin{aligned}
L & =\sum_{i=1}^{m}\left\lceil\log \frac{i+2 n}{\max \left(\operatorname{occ}(s[i], s[1 . . i])-\left\lfloor\log ^{3 / 2} m\right\rfloor, 1\right)}\right\rceil \\
& <\sum_{i=1}^{m} \log (i+2 n)-\sum_{i=1}^{m} \log \max \left(\operatorname{occ}(s[i], s[1 . . i])-\left\lfloor\log ^{3 / 2} m\right\rfloor, 1\right)+m .
\end{aligned}
$$

Since $\{\operatorname{occ}(s[i], s[1 . . i]): 1 \leq i \leq m\}$ and $\{j: 1 \leq j \leq \operatorname{occ}(a, s), a$ a character $\}$ are the same multiset,

$$
\begin{aligned}
L & <\sum_{i=1}^{m} \log (i+2 n)-\sum_{a} \sum_{j=1}^{\mathrm{occ}(a, s)-\left\lfloor\log ^{3 / 2} m\right\rfloor} \log j+m \\
& \leq \sum_{i=1}^{m} \log i+2 n \log (m+2 n)-\sum_{a} \sum_{j=1}^{\mathrm{occ}(a, s)} \log j+n \log ^{5 / 2} m+m \\
& =\log (m !)-\sum_{a} \log (\operatorname{occ}(a, s) !)+m+O\left(n \log ^{5 / 2} m\right) .
\end{aligned}
$$

Therefore, by Stirling's Formula, ${ }^{4}$

$$
\begin{aligned}
L & \leq m \log m-m \ln 2-\sum_{a}(\operatorname{occ}(a, s) \log \operatorname{occ}(a, s)-\operatorname{occ}(a, s) \ln 2)+m+O\left(n \log ^{5 / 2} m\right) \\
& =\sum_{a} \operatorname{occ}(a, s) \log \frac{m}{\operatorname{occ}(a, s)}+m+O\left(n \log ^{5 / 2} m\right) \\
& =(H+1) m+O\left(n \log ^{5 / 2} m\right) .
\end{aligned}
$$

The second line of the above equality uses the fact that $m=\sum \operatorname{occ}((, a))$.

\section{Lower bound}

It is not difficult to show that any prefix coder uses at least $(H+1) m-o(m)$ bits in the worst case (e.g., when $s$ consists of $m-1$ copies of one character and 1 copy of another, so $H m<\log m+\log e$ ). However, this does not rule out the possibility of an algorithm that always uses, say, at most $m / 2$ more bits than static Huffman coding. Vitter [18] proved such a bound is unachievable with an adaptive Huffman coder, and we now extend his result to all adaptive prefix coders. This implies that for an adaptive prefix coder to have a stronger worst-case upper bound than ours (except for lower-order terms), that bound

\footnotetext{
${ }^{4}$ Since $\log (m !)-\sum_{a} \log (\operatorname{occ}(a, s) !)=\log \left(m ! / \prod_{a} \operatorname{occ}(a, s) !\right)$ is the logarithm of the number of ways to arrange the characters in $s$, from this point we could also establish our claim by purely information theoretic arguments.
} 
can be in terms of neither the empirical entropy nor the number of bits used by static Huffman coding. ${ }^{5}$

Theorem 2. Any adaptive prefix coder stores $s$ in at least $m-o(m)$ more bits in the worst case than static Huffman coding.

Proof. Suppose $n=m^{1 / 2}=2^{\ell}+1$ and the first $n$ characters of $s$ are an enumeration of the alphabet. For $n<i \leq m$, when the adaptive prefix coder reaches $s[i]$, there are at least two characters assigned codewords of length at least $\ell+1$; therefore, in the worst case, the coder uses at least $(\ell+1) m-o(m)$ bits. On the other hand, a static prefix coder can assign codewords of length $\ell$ to the $n-2$ most frequent characters and codewords of length $\ell+1$ to the two least frequent ones, and thus use at most $\ell m+o(m)$ bits. Therefore, since a Huffman code minimizes the expected codeword length, any adaptive prefix coder uses at least $m-o(m)$ more bits in the worst case than static Huffman coding.

\section{Online stable sorting}

Consider $s$ as a multiset of characters and suppose we want to sort it stably, online and using only binary comparisons. A stable sort is one that preserves the order of equal elements, and by 'online' we mean every comparison must have the character we read most recently as one of its two arguments. Gagie [6] noted that, by replacing Shannon's construction by a modified construction due to Gilbert and Moore [8], his coder can be used to sort $s$ using $(H+2) m+o(m)$ comparisons and $O(\log n)$ worst-case time for each comparison. We can use our results to speed up Gagie's sorter when $n=o(\sqrt{m / \log m})$.

Whereas Shannon's construction assigns a prefix-free binary codeword of length $\left\lceil\log \left(1 / p_{i}\right)\right\rceil$ to each character with probability $p_{i}>0$, Gilbert and Moore's construction assigns a codeword of length $\left\lceil\log \left(1 / p_{i}\right)\right\rceil+1$. If we take the trie of the codewords, label the leaves from left to right with the characters in the alphabet and label each internal node with the label of the rightmost leaf in its left subtree, the result is a leaf-oriented binary search tree. Building this tree takes $O(n)$ time because, unlike Shannon's construction, we do not need to sort the characters by probability. Hence, although we don't know how to update the alphabetic tree efficiently, we can construct it from scratch in $O(n)$ time. We can apply the same approach as in previous sections, and use searching with delays: while we use the optimal alphabetic tree for $s[1 . . i-n / 2]$ to identify symbols $s[i], s[i+1], \ldots, s[i+n / 2]$, we construct the tree for $s[1 . . i]$ in the background. If we use $O(1)$ time per symbol to construct the next optimal tree, the next tree

\footnotetext{
${ }^{5}$ Notice we do not exclude the possibility of natural probabilistic settings in which our algorithm is suboptimal - e.g., if $s$ is drawn from a memoryless source for which a Huffman code has smaller redundancy than a Shannon code, then adaptive Huffman coding almost certainly achieves better asymptotic compression than adaptive Shannon coding - but in this paper we are interested only in worst-case bounds.
} 
will be completed when $s[i+n / 2]$ is identified. Hence, we identify each character using at most

$$
\left\lceil\log \frac{i+n}{\max (\operatorname{occ}(s[i], s[1 . . i])-n, 1)}\right\rceil+1
$$

comparisons and $O(1)$ worst-case time for each comparison. A detailed description of the algorithm wil be given in the full version of this paper. The following technical lemma, whose proof we omit because it is essentially the same as that of Lemma 4, bounds the total number of comparisons we use to sort $s$ and, thus, implies our speed-up.

\section{Lemma 5.}

$$
\sum_{i=1}^{m}\left\lceil\log \frac{i+n}{\max (\operatorname{occ}(s[i], s[1 . . i])-n, 1)}\right\rceil+m \leq(H+2) m+O\left(n^{2} \log m\right) .
$$

Theorem 3. If $n=o(\sqrt{m / \log m})$, then we can sort s stably and online using $(H+2) m+o(m)$ binary comparisons and $O(1)$ worst-case time for each comparison.

We prove the following theorem by essentially the same arguments as for Theorem 2. It shows that, when $n=o(\sqrt{m / \log m})$, our sorter is essentially optimal. We leave as an open problem finding a sorter that uses $(H+2) m+o(m)$ comparisons and $O(1)$ worst-case time per comparison when $n$ is closer to $m$.

Theorem 4. Any online stable sort uses at least $(H+2) m-o(m)$ binary comparisons in the worst case.

Proof. Suppose $n=m^{1 / 2}=2^{\ell+1}+2, s$ contains only the even-numbered characters in the alphabet, and the first $2^{\ell}+1$ characters of $s$ are an enumeration of those characters. It is not difficult to show that, for $2^{\ell}+1<i \leq m$, the online stable sorter must determine the identity of $s[i]$, in case it is an odd-numbered character; moreover, it must do this before reading $s[i+1]$, in case all the remaining characters in $s$ are smaller than $s[i]$ 's predecessor in $\{s[j]: 1 \leq j<i\}$ or larger than its successor. Therefore, after $s\left[2^{\ell}+1\right]$, we can view the sorter as processing each character using a binary decision tree with $n$ leaves, labelled from left to right with the characters in the alphabet in lexicographic order. (Since we are proving a worst-case lower bound, we assume without loss of generality that the sorter is deterministic.) For $2^{\ell}+1<i \leq m$, when the sorter reaches $s[i]$, there must be a leaf at depth at least $\ell+2$ that is labelled with an even-numbered character in the alphabet. Therefore, even when $s$ contains only the even-numbered characters in the alphabet, the sorter uses at least $(\ell+2) m-o(m)$ comparisons in the worst case. Since $s$ contains only $2^{\ell}+1$ distinct characters, however, $H \leq \log \left(2^{\ell}+1\right)$ and calculation shows $(H+2) m-o(m) \leq(\ell+2) m-o(m)$. 


\section{Other Coding Problems}

Several variants of the prefix coding problem were considered and extensively studied. In the alphabetic coding problem [8], codewords must be sorted lexicographically, i.e. $i<j \Rightarrow c\left(a_{i}\right)<c\left(a_{j}\right)$, where $c\left(a_{k}\right)$ denotes the codeword of $a_{k}$. In the length-limited coding problem, the maximum codeword length is limited by a parameter $F>\log n$. In the coding with unequal letter costs problem, one symbol in the code alphabet costs more than another and we want to minimize the average cost of a codeword. All of the above problems were studied in the static scenario. Adaptive prefix coding algorithms for those problems were considered in [5]. In this section we show that the good upper bounds on the length of the encoding can be achieved by algorithms that encode in $O(1)$ worst-case time. The main idea of our improvements is that we encode a symbol $s[i]$ in the input string with a code that was constructed for the prefix $s[1 . . i-d]$ of the input string, where the parameter $d$ is chosen in such a way that a corresponding (almost) optimal code can be constructed in $O(d)$ time. Using the same arguments as in the proof of Lemma 4 we can show that encoding with delays increases the length of encoding by an additive term of $O(d \cdot n \log m)$ (the analysis is more complicated in the case of coding with unequal letter costs). We will provide proofs in the full version of this paper.

Alphabetic Coding. The algorithm of section 6 can be used for adaptive alphabetic coding. The length of encoding is $((H+2) m+o(m)$ provided that $n=o(\sqrt{m / \log m})$. Unfortunately we cannot use the encoding and decoding methods of section 2 because the alphabetic coding is not canonical. When an alphabetic code for the following group of $O(n)$ symbols is constructed, we also create in $O(n)$ time a table that stores the codeword of each symbol $a_{i}$. Such a table can be created from the alphabetic tree in $O(n)$ time; hence, the complexity of the encoding algorithm is not increased. We can decode the next codeword by searching in the data structure that contains all codewords. Using a data structure due to Andersson and Thorup [2] we can decode in $O(\min (\sqrt{\log n}, \log \log m)$ time per symbol.

Theorem 5. There is an algorithm for adaptive alphabetic prefix coding that encodes and decodes each symbol of a string s in $O(1)$ and $O(\min (\sqrt{\log n}, \log \log m))$ time respectively. If $n=o(\sqrt{m / \log m})$, the encoding length is $((H+2) m+o(m)$.

Coding with unequal letter costs. Krause [12] showed how to modify Shannon's construction for the case in which code letters have different costs, e.g., the different durations of dots and dashes in Morse code. Consider a binary channel and suppose $\operatorname{cost}(0)$ and $\operatorname{cost}(1)$ are constants with $0<\operatorname{cost}(0) \leq \operatorname{cost}(1)$. Krause's construction gives a code such that, if a symbol has probability $p$, then its codeword has cost less than $\ln (p) / C+\operatorname{cost}(1)$, where the channel capacity $C$ is the largest real root of $e^{-\operatorname{cost}(0) \cdot x}+e^{-\operatorname{cost}(1) \cdot x}=1$ and $e$ is the base of the natural logarithm. It follows that the expected codeword cost in the resulting code is $H \ln 2 / C+\operatorname{cost}(1)$, compared to Shannon's bound of $H \ln 2 / C$. Based on Krause's construction, Gagie gave an algorithm that produces an encoding of $s$ with total cost at most $\left(\frac{H \ln 2}{C}+\operatorname{cost}(1)\right) m+o(m)$ in $O(m \log n)$ time. Since the 
code of Krause [12] can be constructed in $O(n)$ time, we can use the encoding with delay $n$ and achieve $O(1)$ worst-case time. Since the costs are constant and the minimum probability is $\Omega(1 / m)$, the maximum codeword length is $O(\log m)$. Therefore, we can decode using the data structure described above.

Theorem 6. There is an algorithm for adaptive prefix coding with unequal letter costs that encodes and decodes each symbol of a string $\sin O(1)$ and $O(\min (\sqrt{\log n}, \log \log m))$ time respectively. If $n=o(\sqrt{m / \log m})$, the encoding length is $\left(\frac{H \ln 2}{C}+\operatorname{cost}(1)\right) m+$ $o(m)$.

Length-limited coding. Finally, we can design an algorithm for adaptive length-limited prefix coding by modifying the algorithm of section 4 . Using the same method as in [5] - i.e., smoothing the distribution by replacing each probability with a weighted average of itself and $1 / n$ - we set the codeword length of symbol $s[i]$ to $\left\lceil\log \frac{2^{f}}{\left(2^{f}-1\right) x+1 / n}\right\rceil$ instead of $\left\lceil\log \frac{1}{x}\right\rceil$, where $x=\frac{\max \left(\operatorname{occ}(s[i], s[1 . . i])-\left\lfloor\log ^{3 / 2} m\right\rfloor, 1\right)}{i+2 n}$ and $f=F-\log n$. We observe that the codeword lengths $l_{i}$ of this modified code satisfy the Kraft-McMillan inequality:

$$
\begin{aligned}
\sum_{i} 2^{-l_{i}} & \leq \sum_{x}\left(\left(2^{f}-1\right) x+1 / n\right) / 2^{f} \\
& =\sum_{x}\left(2^{f} x\right) / 2^{f}-\sum_{x} x / 2^{f}+n(1 / n) / 2^{f}=1
\end{aligned}
$$

Therefore we can construct and maintain a canonical prefix code with codeword lengths $l_{i}$. Since $\frac{2^{f}}{\left(2^{f}-1\right) x+1 / n} \leq \min \left(\frac{2^{f}}{\left(2^{f}-1\right) x}, \frac{2^{f}}{1 / n}\right),\left\lceil\log \frac{2^{f}}{\left(2^{f}-1\right) x+1 / n}\right\rceil \leq$ $\min \left(\left\lceil\log \frac{2^{f}}{\left(2^{f}-1\right) x}\right\rceil, \log n+f\right)$.. Thus the codeword length is always smaller than $F$. We can estimate the encoding length by bounding the first part of the above expression: $\left\lceil\log \frac{2^{f}}{\left(2^{f}-1\right) x}\right\rceil<x+1+\log \frac{2^{f}+1}{2^{f}}$ and $\log \frac{2^{f}+1}{2^{f}}=\left(1 / 2^{f}\right) \log \left(1+\frac{1}{2^{f}}\right)^{2^{f}} \leq$ $\frac{1}{2^{f} \ln 2}$. Summing up by all symbols $s[i]$, the total encoding length does not exceed

$$
\sum_{i=1}^{m} \log \frac{i+2 n}{\max \left(\operatorname{occ}(s[i], s[1 . . i])-\left\lfloor\log ^{3 / 2} m\right\rfloor, 1\right)}+m+\frac{m}{2^{f} \ln 2} .
$$

We can estimate the first term in the same way as in Lemma 4 ; hence, the length of the encoding is $\left(H+1+\frac{1}{2^{f} \ln 2}\right) m+O\left(n \log ^{5 / 2} m\right)$. We thus obtain the following theorem:

Theorem 7. There is an algorithm for adaptive length-limited prefix coding that encodes and decodes each symbol of a string $s$ in $O(1)$ time. The encoding length is $\left(H+1+\frac{1}{2^{f} \ln 2}\right) m+O\left(n \log ^{5 / 2} m\right)$, where $f=F-\log n$ and $F$ is the maximum codeword length.

\section{References}

1. A. Andersson, P. Bro Miltersen, and M. Thorup. Fusion trees can be implemented with $\mathrm{AC}^{0}$ instructions only. Theoretical Computer Science, 215(1-2):337-344, 1999. 
2. A. Andersson and M. Thorup. Dynamic ordered sets with exponential search trees. Journal of the ACM, 54(3), 2007.

3. N. Faller. An adaptive system for data compression. In Record of the 7th Asilomar Conference on Circuits, Systems and Computers, pages 593-597, 1973.

4. M. L. Fredman and D. E. Willard. Surpassing the information theoretic bound with fusion trees. Journal of Computer and System Sciences, 47(3):424-436, 1993.

5. T. Gagie. Dynamic Shannon coding. In Proceedings of the 12th European Symposium on Algorithms, pages 359-370, 2004.

6. T. Gagie. Dynamic Shannon coding. Information Processing Letters, 102(2-3):113$117,2007$.

7. R. G. Gallager. Variations on a theme by Huffman. IEEE Transactions on Information Theory, 24(6):668-674, 1978.

8. E. N. Gilbert and E. F. Moore. Variable-length binary encodings. Bell System Technical Journal, 38:933-967, 1959.

9. D. A. Huffman. A method for the construction of minimum-redundancy codes. Proceedings of the IRE, 40(9):1098-1101, 1952.

10. M. Karpinski and Y. Nekrich. A fast algorithm for adaptive prefix coding. Algorithmica, to appear.

11. D. E. Knuth. Dynamic Huffman coding. Journal of Algorithms, 6(2):163-180, 1985.

12. R. M. Krause. Channels which transmit letters of unequal durations. Information and Control, 5(1):13-24, 1962.

13. R. L. Milidiú, E. S. Laber, and A. A. Pessoa. Bounding the compression loss of the FGK algorithm. Journal of Algorithms, 32(2):195-211, 1999.

14. A. Moffat and A. Turpin. On the implementation of minimum redundancy prefix codes. IEEE Transactions on Communications, 45(10):1200-1207, 1997.

15. E. S. Schwartz and B. Kallick. Generating a canonical prefix encoding. Communications of the ACM, 7(3):166-169, 1964.

16. C. E. Shannon. A mathematical theory of communication. Bell System Technical Journal, 27:379-423, 623-656, 1948.

17. A. Turpin and A. Moffat. On-line adaptive canonical prefix coding with bounded compression loss. IEEE Transactions on Information Theory, 47(1):88-98, 2001.

18. J. S. Vitter. Design and analysis of dynamic Huffman codes. Journal of the ACM, 1987(4):825-845, 1987. 